\title{
Pulmonary Involvement in Neonatal Lupus from Clinical Diagnoses to Autopsy Findings: A Case Report
}

\section{Fan Wu ( $\sim$ gdwufan@126.com )}

Guangzhou Medical University https://orcid.org/0000-0002-8564-4619

\section{Li Ying}

Guangzhou Medical University

\section{Xiong Hanzhen}

Guangzhou Medical University

Lin Lili

Guangzhou Medical University

\section{Huang Weiliang}

Guangzhou Medical University

\section{Case report}

Keywords: Neonatal lupus erythematosus, Acute lupus pneumonitis

Posted Date: October 11th, 2021

DOl: https://doi.org/10.21203/rs.3.rs-951370/v1

License: (c) (1) This work is licensed under a Creative Commons Attribution 4.0 International License. Read Full License 


\section{CASE REPORT}

\section{Pulmonary involvement in neonatal lupus from clinical diagnoses to autopsy findings: A case report}

Li Ying ${ }^{1,2 \dagger}$, jia Chunhong ${ }^{1,2^{\dagger}}$, Xiong Hanzhen ${ }^{3}$, Lin Lili $^{1}$, Huang Weiliang ${ }^{1}$, Chen ruiqi $^{1}$, Wu Fan $^{1,2 *}$

${ }^{1}$ Department of Pediatrics, the Third Affiliated Hospital of Guangzhou Medical University, Guangzhou, Guangdong 510150, China

${ }^{2}$ Key Laboratory for Major Obstetric Disease of Guangdong Province, Guangzhou, Guangdong 510150, China

${ }^{3}$ Department of Pathology, the Third Affiliated Hospital of Guangzhou Medical University, Guangzhou, Guangdong 510150, China

* Correspondence: gdwufan@126.com

$\dagger$ Li Ying and Jia Chunhong contributed equally to this work. 


\begin{abstract}
Background: Neonatal lupus erythematosus (NLE) is an uncommon immunemediated disease caused by the transplacental passage of maternal autoantibodies. In this paper, we described a case of a newborn with NLE involving lung damage from clinical diagnoses to autopsy findings.

Case presentation: The mother had no history of immune system disease; however, the test for antinuclear antibodies was positive. A boy was delivered spontaneously at 36+2 weeks' gestation without asphyxia (birth weight: $1.21 \mathrm{~kg}$, length: $38 \mathrm{~cm}$, head circumference: $26 \mathrm{~cm}$ ). Soon after birth, intermittent shortness of breath occurred, followed by cyanosis and severe hypoxemia. Blood tests suggested that the infant was positive for anti-nuclear antibodies. Although multiple respiratory support methods were used, death could not be avoided and occurred at 6.5 days due to cardiopulmonary failure. The results of the autopsy revealed diffuse interstitial inflammation and interstitial fibrosis in both lungs, manifesting as telangiectasia, hyperemia, alveolar cavity dilatation and fusion, and obvious interstitial widening with chronic inflammatory cell infiltration. The newborn was finally diagnosed with nonspecific interstitial pneumonia with fibrosis caused by congenital systemic lupus erythematosus.
\end{abstract}

Conclusions: Pulmonary involvement with NLE is a diagnostic challenge at present. Close attention should be given to newborns whose mothers have systemic lupus erythematosus.

\title{
Keywords
}

Neonatal lupus erythematosus, Acute lupus pneumonitis

\section{Introduction}

Neonatal lupus erythematosus (NLE) is a very rare disease of the developing fetus and neonate that is related to the transplacental passage of maternal autoantibodies(1). In approximately $95 \%$ of cases, autoantibodies against Sjogren syndrome A (SSA/Ro), Sjogren syndrome B (SSB/La), or rarely U1RNP in the mother are positive. Specifically, half of affected infants are born to healthy mothers whose seropositivity is discovered after the infant is diagnosed(2-4). The others often have mothers who are diagnosed with rheumatic conditions, such as systemic lupus erythematous (SLE)(3). NLE infants rarely have negative SSA/Ro antibody tests, but in such cases, NLE infants may have other autoantibodies (SSB/La and U1RNP)(5). NLE is considered to be a form of passively acquired autoimmunity. 
The most common clinical manifestations of NLE are observed in the skin, heart, blood, liver, nerve and another organ alone or in combination. Pulmonary involvement is rare in newborns. In a few cases, pneumonitis was transient or occurred in the setting of cardiac disease(6). Here, we present a case of acute lupus pneumonitis in a newborn with NLE that clinically manifested as respiratory failure, and autopsy results suggested nonspecific interstitial pneumonia with fibrosis. We hope that this case will contribute to revealing the pathology of pulmonary involvement with NLE and facilitate a timely diagnosis.

\section{Case presentation}

The mother, a 29-year-old primigravida, had no family history of autoimmune system diseases. However, positive antinuclear antibodies (ANAs) in serum were reported at $24^{+3}$ weeks' gestation, and treatment was started at $33^{+3}$ weeks gestation (200 mg/bid hydroxychloroquine sulfate, $20 \mathrm{mg}$ qam methylprednisolone, $50 \mathrm{mg}$ bid cyclosporine). The patient was still positive for ANAs at $35^{+5}$ weeks' gestation: karyotype 1 titer (> 1:1000), karyotype 2 titer (>1:1000), anti-nuclear antibody (349.562 U/ml), and positive SSA/Ro-52, SSA/Ro-60, and SSB. A boy was delivered spontaneously at $36^{+2}$ weeks' gestation without asphyxia (birth weight: $1.21 \mathrm{~kg}$, length: $38 \mathrm{~cm}$, head circumference: $26 \mathrm{~cm}$ ).

Due to premature and SGA the boy was admitted to the neonatal intensive care unit (NICU). Except for patent ductus arteriosus $(2.1 \mathrm{~mm})$ and atrial septal defects (1.4 $\mathrm{mm}$ ), there were no abnormalities found. The electrocardiogram (ECG) monitoring seems normal and similar for the chest X-ray radiograph on the first $24 \mathrm{~h}, 63 \mathrm{~h}$ and even at the time of death (Figure 1A-1C). However, blood tests showed that hs-CRP increased to $26.24 \mathrm{mg} / \mathrm{L}$ at $37 \mathrm{~h}$ and $35 \mathrm{mg} / \mathrm{L}$ at $63 \mathrm{~h}$, and then decreased to $15.1 \mathrm{mg} / \mathrm{L}$ following treatment with latamoxef or meropenem + linezolid. More seriously, intermittent shortness of breath occurred at $58 \mathrm{~h}$, and dyspnea further worsened manifested as cyanosis at $63 \mathrm{~h}\left(\mathrm{pH} 7.27, \mathrm{FiO}_{2} 21 \%, \mathrm{PCO}_{2} 41 \mathrm{mmHg}, \mathrm{PO}_{2} 51 \mathrm{mmHg}\right.$, $\mathrm{BE}-7.2 \mathrm{mmol} / \mathrm{L}, \mathrm{SaO}_{2} 81 \%$ ) and irreformable hypoxemia $\left(\mathrm{pH} 7.32, \mathrm{FiO}_{2} 40 \%, \mathrm{PCO}_{2}\right.$ $48 \mathrm{mmHg}, \mathrm{PO}_{2} 30 \mathrm{mmHg}, \mathrm{BE}-1.4 \mathrm{mmol} / \mathrm{L}, \mathrm{SaO}_{2} 50.6 \%$ ). With the aggravation of symptoms, the continuous positive airway pressure, mechanical ventilation under endotracheal intubation and high-frequency invasive mechanical ventilation was started were successively adopted. However, severe hypoxemia occurred at 114 hours (pH 7.31, $\mathrm{FiO}_{2}$ 100\%, $\mathrm{PCO}_{2} 45 \mathrm{mmHg}, \mathrm{PO}_{2} 26 \mathrm{mmHg}, \mathrm{BE}-3.2 \mathrm{mmol} / \mathrm{L}, \mathrm{SaO}_{2}$ $42.3 \%$ ) and laboratory examination indicated pulmonary hypertension. Subsequently, alprostadil (10 $\mathrm{ng} / \mathrm{kg} \cdot \mathrm{min})$ and hydrocortisone $(1 \mathrm{mg} / \mathrm{kg}, \mathrm{q} 8 \mathrm{~h})$ were administered on the 6th day. Unfortunately, the baby suffered from refractory hypoxemia, and cardiopulmonary failure occurred on day 6.5. Blood gas analysis indicated type II respiratory failure $\left(\mathrm{pH}\right.$ 6.52, $\mathrm{FiO}_{2} 100 \%, \mathrm{PCO}_{2} 106$ mmHg, $\mathrm{PO}_{2} 35 \mathrm{mmHg}, \mathrm{BE}-26.7$ 
$\left.\mathrm{mmol} / \mathrm{L}, \mathrm{SaO}_{2} 17.2 \%\right)$. The day he died, the ANA test was positive: karyotype 1 titer (1:3200), anti-nuclear antibody (334.39 U/ml), positive SSA/Ro-52, SSA/Ro-60, and SSB and negative anti-double-stranded DNA.

With the consent of his parents, an autopsy was performed to further clarify the disease. The results indicated that there were no obvious abnormalities in the head, neck, thorax, digestive tract, spleen, pancreas, liver biliary system, urinary system, genitals or vertebral column limb. A piece of skin tissue was taken for histological analysis. HE staining showed no significant pathological changes (Figure 2A), but immunofluorescence staining suggested $\operatorname{IgG}(+), \operatorname{IgA}(-), \operatorname{IgM}(+), C 3(+)$, and C1q (perivascular granular deposition), The immunofluorescence of IgG was shown in Figure 2B. Diffuse interstitial fibrosis with inflammatory cell infiltration, telangiectasia and congestion was noted in lung tissue. Additionally, the alveolar septum was widened, the alveolar cavity was enlarged, and some of the alveoli were ruptured and fused (Figure 3A). Immunofluorescence staining revealed $\operatorname{IgG}(+), \operatorname{IgA}$ (-), IgM (alveolar wall +), C3 (focal alveolar wall deposition), and C1q (alveolar interstitial scattered granular deposition), and the immunofluorescence of $\mathrm{IgG}$ was shown in Figure 3B. Although the appearance of the heart and lung was normal, the heart was slightly enlarged, and the ventricular wall was thickened (Figure 3C and 3D).

\section{Discussion}

NLE is a rare neonatal immune disease. Maternal antibodies such as anti-SSA or anti-SSB antibodies are transferred to the fetus via the placenta and result in damage to fetal tissues $(7,8)$. However, the precise mechanism has not been clarified. NLE presents with reversible manifestations, including cutaneous lesions in $40 \%$ of the cases, hepatic dysfunction in $35 \%$ of cases, and hematologic abnormalities in $35 \%$ of cases. Twenty-five percent of neonates present with irreversible cardiac arrhythmias, the most distinctive and severe feature of NLE(9). Previous studies have shown that most of the newborns born to mothers with SLE are rarely diagnosed in less than a week, and most of them have a good prognosis. There are few reports of infant death, especially neonatal death. Death cases are often infants suffering from irreversible cardiac arrhythmias(2). There is a small number of reports of NLE-related pneumonitis. In those cases, pneumonitis was transient or occurred in the setting of cardiac disease(6). In our case, the infant who survived less than a week presented with progressive and uncorrectable respiratory distress without irreversible cardiac arrhythmias or rash or hepatic dysfunction. Although there was an increase in hs-CRP levels in the early stage of the disease, the chest X-ray did not show any signs of pneumonia. After treatment with antibiotics and mechanical ventilation, although the hs-CRP level decreased considerably, the newborn still had progressive pulmonary 
hypertension and hypoxemia, which were difficult to correct and ultimately caused his death. Therefore, the respiratory distress may not be caused by neonatal pneumonitis.

The autopsy results showed nonspecific interstitial pneumonia with fibrosis. Immunofluorescence staining showed IgG (+), IgA (-), IgM (alveolar wall +), C3 (focal alveolar wall deposition), and C1q (alveolar interstitial scattered granular deposition) in the lung tissue. As reported in previous studies, the pathology of NLE is involved in acute lupus pneumonitis (ALP), interstitial pneumonitis, hyaline membrane formation, alveolar necrosis, edema, microvascular thrombosis and focal polymorphonuclear infiltration without evidence of vasculitis(10). Based on the clinical symptoms and results of the autopsy, ALP was confirmed, which was the leading cause of refractory pulmonary hypertension and death. ALP has been reported in infants with no apparent infection and may resolve or progress to chronic interstitial pneumonitis, which is consistent with our case $(11,12)$. Pulmonary manifestations may be a life-threatening complication. In a case series of ALP as a presentation of SLE, the mortality rate was $40 \%$ (11). Referring to previous case reports, the current treatment of LP uses mainly high-dose steroids. Other immunosuppressive drugs, such as azathioprine, cyclophosphamide, and intravenous immunoglobulin, may be used for severe cases that are refractory to corticosteroids $(13,14)$. Unfortunately, due to the rapid progression of the disease and the short survival time, the treatment mentioned above has not been implemented. Although this report presents a case of neonatal failure to survive, it is the first to describe the results of an autopsy of a newborn who died early in NLE and to confirm ALP at the pathophysiological level.

Pulmonary involvement with NLE is a diagnostic challenge at present. The neonate may have no obvious symptoms at birth, and once the disease occurs, the disease progresses very quickly and is even life-threatening. Close attention should be given to newborns whose mothers have systemic lupus erythematosus.

\section{Abbreviations}

NLE: Neonatal lupus erythematosus; SSA/Ro: Sjogren syndrome A; SSB/La: Sjogren syndrome B; SLE: systemic lupus erythematous; ANAs: antinuclear antibodies; NICU: neonatal intensive care unit; ECG: electrocardiogram; ALP: acute lupus pneumonitis

\section{Declarations}

\section{Ethics approval and consent to participate}


This case report was performed in accordance with institutional ethics regulations.

\section{Consent for participate}

Consent for publication Written informed consent was obtained from the patient's parents for publication of this case report including clinical data and any accompanying images.

\section{Availability of data and materials}

All data generated or analyzed during this study are included in this published article.

\section{Competing interests}

The authors declare that they have no competing interests.

\section{Funding}

This work was supported by Guangdong Science, Technology Grants (2021A1515011225 to WF), Science and Technology Planning Project of Guangzhou (202102010080 to WF)

\section{Authors' contributions}

Ying Li, Fan Wu designed and operated the project. Chunhong Jia and Hanzhen Xiong provided the radiological and pathological analyses. Chunhong Jia, Lili Lin Huang Weiliang and Chen ruiqi support the literature search and text proofreading. All authors read and approved the final manuscript.

\section{Acknowledgements}

We appreciate the patient parents for their gracious participation and support.

\section{References}

1. Vanoni F, Lava SAG, Fossali EF, Cavalli R, Simonetti GD, Bianchetti MG, et al. Neonatal Systemic Lupus Erythematosus Syndrome: a Comprehensive Review. Clin Rev Allergy Immunol. 2017;53(3):46976.

2. Heelan K, Watson R, Collins SM. Neonatal lupus syndrome associated with ribonucleoprotein antibodies. Pediatr Dermatol. 2013;30(4):416-23.

3. Johnson B. Overview of neonatal lupus. J Pediatr Health Care. 2014;28(4):331-41.

4. Zuppa AA, Riccardi R, Frezza S, Gallini F, Luciano RM, Alighieri G, et al. Neonatal lupus: Follow-up in infants with anti-SSA/Ro antibodies and review of the literature. Autoimmun Rev. 2017;16(4):427-

32. 
5. Wozniacka A, Robak E, Sysa-Jedrzejowska A, McCauliffe DP. Pneumocystis carinii mimicking neonatal lupus erythematosus-related pneumonitis. Pediatr Dermatol. 2003;20(6):524-8.

6. Morton RL, Moore C, Coventry S, Langston C, Schikler K, Eid NS. Pulmonary capillaritis and hemorrhage in neonatal lupus erythematosus (NLE). J Clin Rheumatol. 2004;10(3):130-3.

7. Lee LA. The clinical spectrum of neonatal lupus. Arch Dermatol Res. 2009;301(1):107-10.

8. Gleicher N, Elkayam U. Preventing congenital neonatal heart block in offspring of mothers with anti-SSA/Ro and SSB/La antibodies: a review of published literature and registered clinical trials. Autoimmun Rev. 2013;12(11):1039-45.

9. Chen CC, Huang JL, Hsu JF, Chung CY, Lin KL. Neonatal lupus complicated by hemorrhagic stroke. Lupus. 2012;21(14):1582-5.

10. Wu J, Berk-Krauss J, Glick SA. Neonatal Lupus Erythematosus. JAMA Dermatol. 2021;157(5):590.

11. Ciftci E, Yalcinkaya F, Ince E, Ekim $M$, lleri $M$, Orgerin Z, et al. Pulmonary involvement in childhood-onset systemic lupus erythematosus: a report of five cases. Rheumatology (Oxford). 2004;43(5):587-91.

12. Silverman E, Jaeggi E. Non-cardiac manifestations of neonatal lupus erythematosus. Scand J Immunol. 2010;72(3):223-5.

13. Chetan G, Mahadevan S, Sulanthung K, Narayanan P. Intravenous immunoglobulin therapy of lupus pneumonitis. Indian J Pediatr. 2007;74(11):1032-3.

14. Wan SA, Teh CL, Jobli AT. Lupus pneumonitis as the initial presentation of systemic lupus erythematosus: case series from a single institution. Lupus. 2016;25(13):1485-90. 
Figure legends

Figures 1 The chest and abdominal X-ray images. The chest radiograph on the first day (A), at $58 \mathrm{~h}(\mathrm{~B})$ and on the 6th day (C) after birth.

Figure 2 Histological analysis for skin tissue. (A) HE staining. (B)

immunofluorescence staining: $\operatorname{IgG}(+)$ particles $(\leftarrow)$ were deposited around the blood vessels.

Figure 3 Histological analysis for lung and heart. (A) HE staining for lung tissue. (B) immunofluorescence staining for lung tissue: $\operatorname{IgG}(+)$ particles $(\leftarrow)$ were scattered in alveolar interstitial tissue. (C) the appearance of lung tissue. (D) the gross anatomy for heart. 
Figures

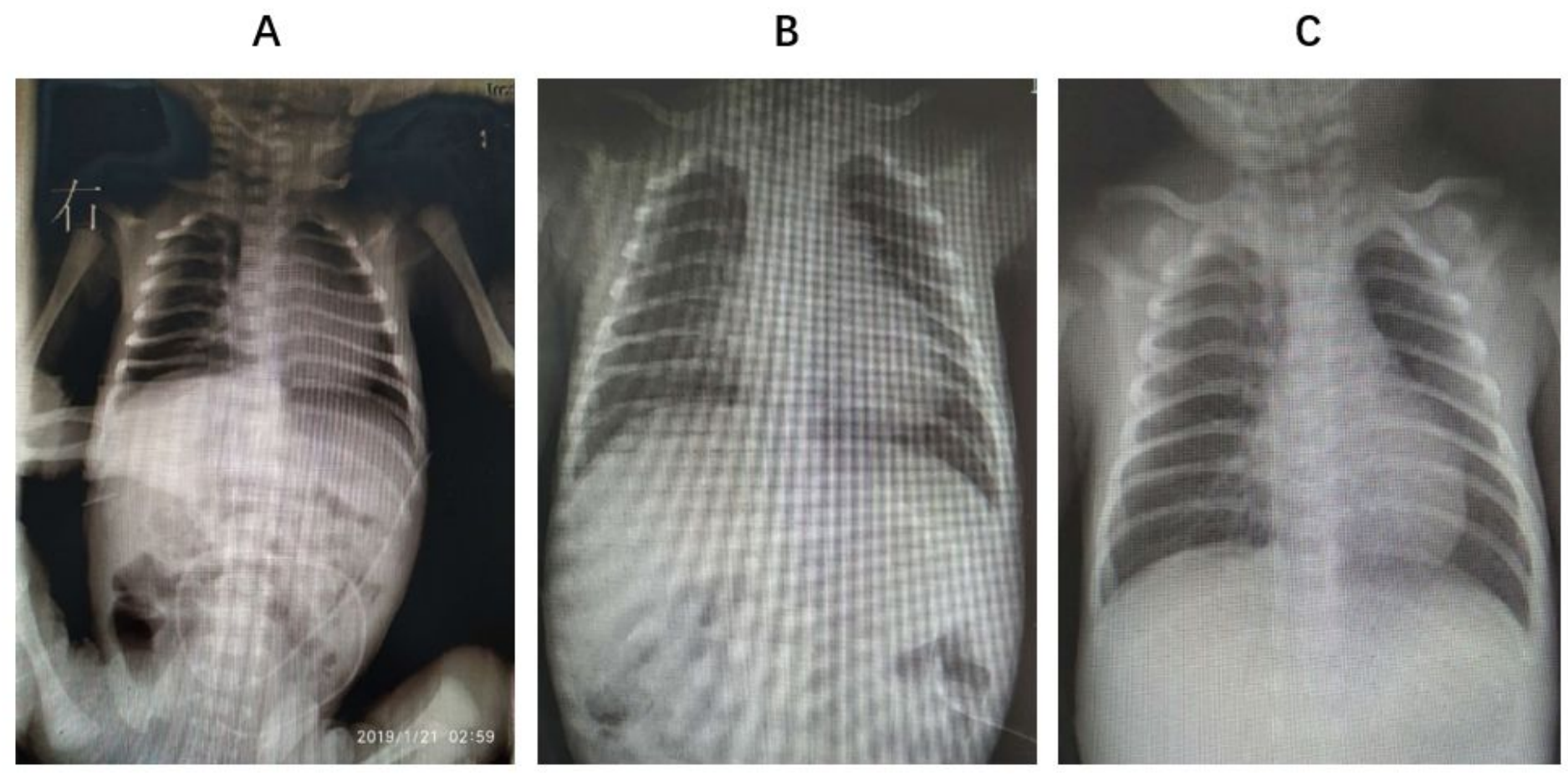

Figure 1

The chest and abdominal X-ray images. The chest radiograph on the first day (A), at $58 \mathrm{~h}(\mathrm{~B})$ and on the 6th day (C) after birth.

A

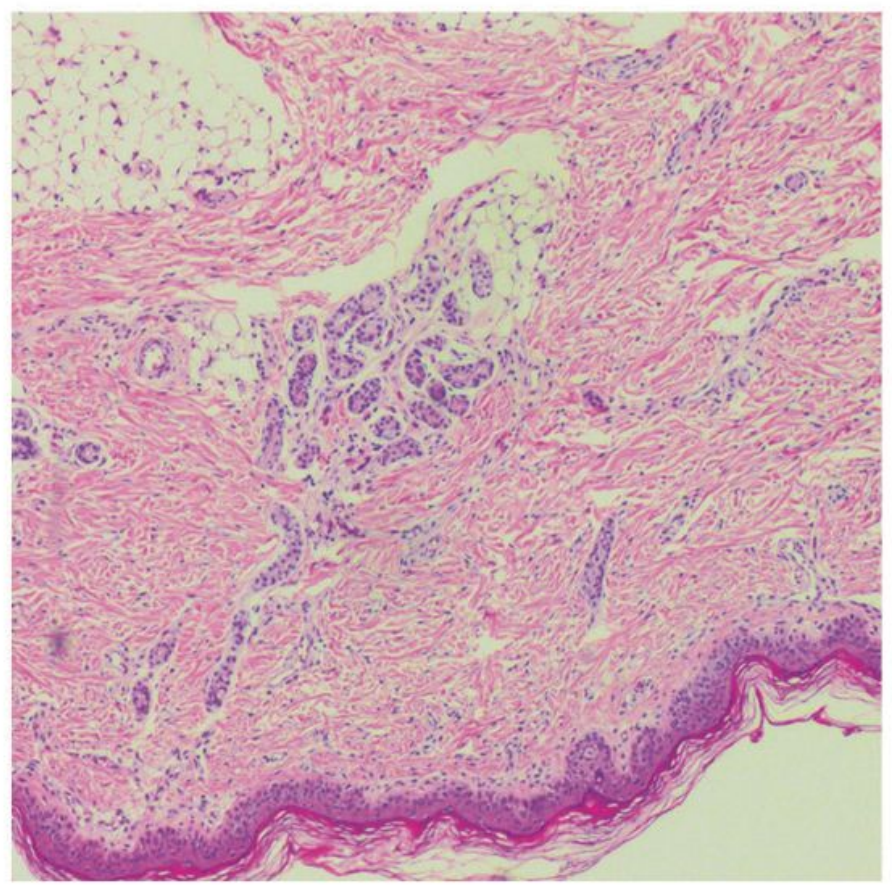

B

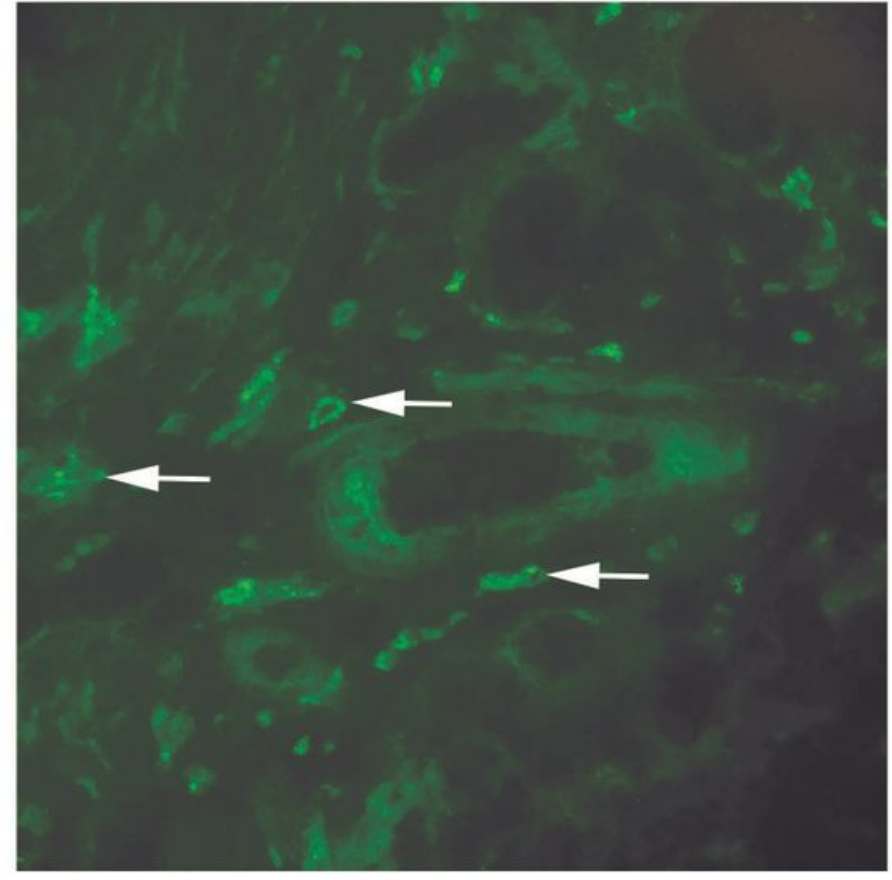

Figure 2 
Histological analysis for skin tissue. (A) HE staining. (B) immunofluorescence staining: IgG (+) particles $(\Downarrow)$ were deposited around the blood vessels.

A

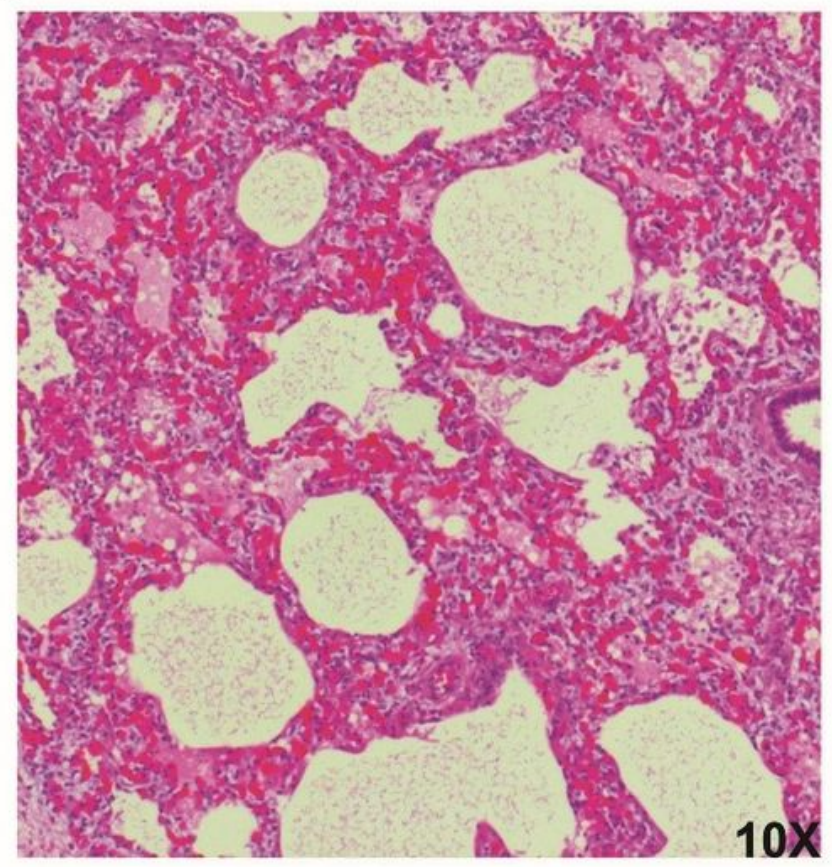

C

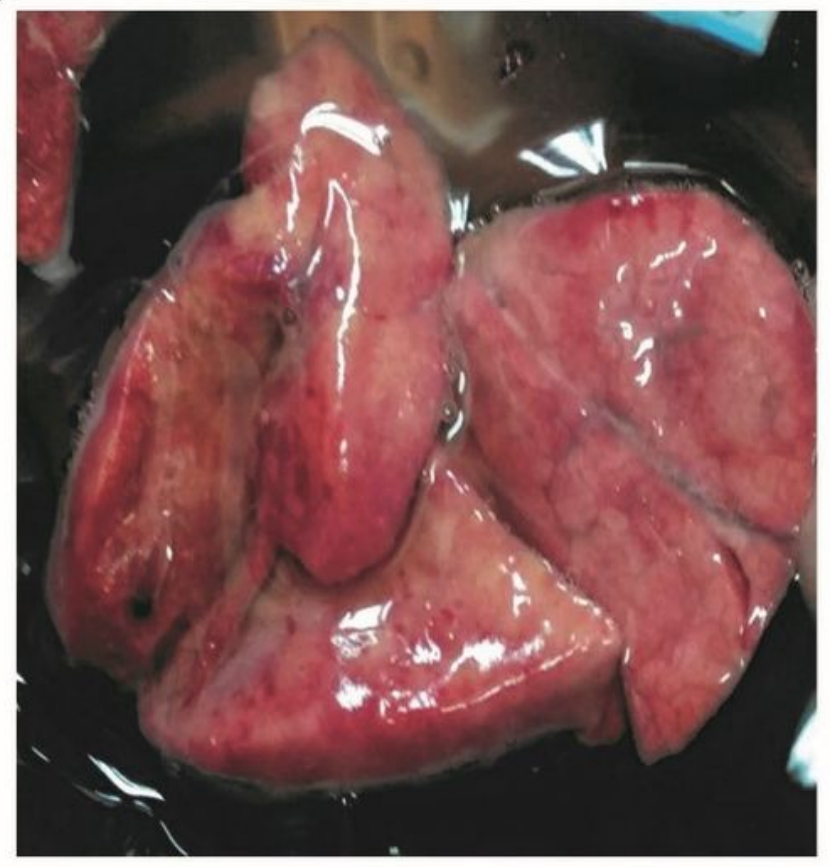

B

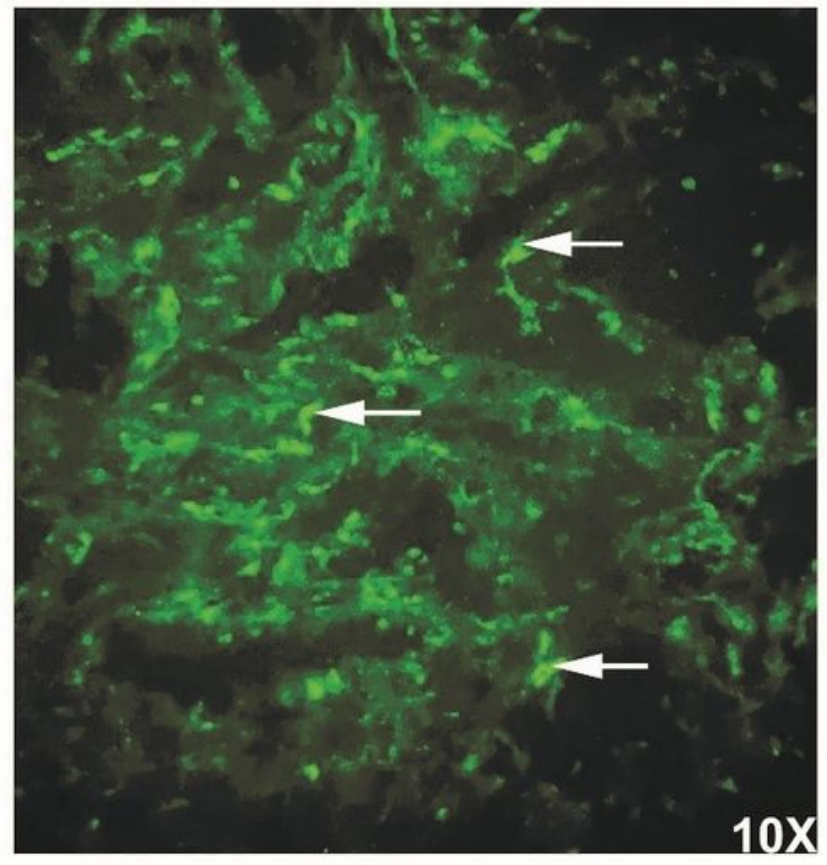

D

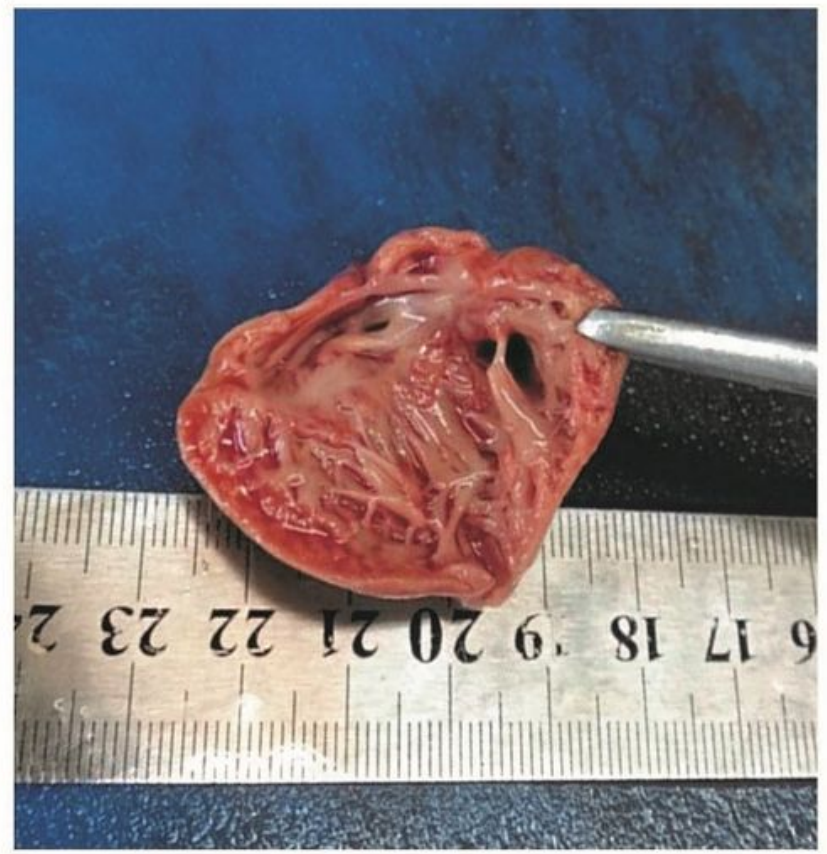

\section{Figure 3}

Histological analysis for lung and heart. (A) HE staining for lung tissue. (B) immunofluorescence staining for lung tissue: IgG (+) particles ( () ) were scattered in alveolar interstitial tissue. (C) the appearance of lung tissue. (D) the gross anatomy for heart. 